\section{LUMINESCENCE AND COLOUR}

A FEW years ago luminescence was little more
to-day it forms the basis of new and important
industries. The Colour Group of the Physical Society
devoted its tenth science meeting, held on Decem-
ber 11, to its consideration, when four papers were
read and discussed. In the opening paper, on "The Chemical and Physical Properties of Luminescent Materials", Dr. J. W. Strange reviewed the development of luminescence and referred to the alchemical, if not culinary, flavour which still pervades the preparation of phosphors. He declared, furthermore, that the lack of rigid chemical control and co-operation between chemist and physicist has accounted for a good deal of the lack of progress in understanding luminescent phenomena. The words alchemical and culinary might, with some justice, be applied to the early attempts at making luminescent powders, but can scarcely be applied to the preparation of modern highly efficient phosphors, where chemical control is rigid in the extreme. So-called pure chemicals as purchased are rarely good enough for manufacturing luminescent powders, and these are generally subjected to careful purification before use.

It is true that theory lags far behind the practice of luminescence, and there does not appear to be much common ground as yet where the theoretical chemist or physicist and the luminescent powder chemist can meet. Nevertheless, as pointed out in the discussion following the meeting, acknowledgment must be made for the contribution of X-ray analysis to the development and understanding of crystalline phosphors. The way in which X-ray spectroscopy has contributed to the elucidation of luminescent problems has recently been described by Rooksby (Electrical Times, 102, 260; 1942).

Dr. Strange described the preparation of crystalline phosphors by firing the precipitated compound or the component oxides with the appropriate activating metal and flux at temperatures between $850^{\circ}$ and $1,200^{\circ} \mathrm{C}$. Manganese is the most important activator, but copper, silver, bismuth and a few others are also used, there being an optimum concentration for each phosphor.

Phosphors such as calcium and magnesium tungstates and zinc sulphide, which do not require an activator in the accepted sense, appear, at first sight, to conflict with the theory of active centres, which is largely based on the discovery that traces of certain heavy metals in a suitable crystalline matrix produce luminescence. The modern tendency, however, is to regard these apparent exceptions as conforming to the general rule. Thus there is evidence that in pure luminescent zine sulphide there is a stoichiometric excess of zinc in the lattice which behaves as the activator. The main function of the flux, which is generally a halide, is to speed up the reaction or to bring it about at a lower temperature. Except possibly in certain sulphide phosphors, which require the presence of a halide for maximum efficiency, the flux does not appear to enter into the composition of the luminescent material.

Dr. Strange referred to some of the uses of luminescence in analytical work, atomic physics, electric discharge tubes and cathode ray tubes. In cathode ray tubes the relation between light output and tube current is of particular interest in connexion with theories of the mechanism of luminescence. It is a remarkable fact that the phosphor still continues to show an increase in light output with increase in current even at a power loading as high as 12 watts per sq. cm. of screen.

$\mathrm{He}$ concluded his remarks with a brief review of the modern interpretation of luminescence based on the idea that the atoms of the activator form the active centres for absorption and emission of radiation.

Mr. H. G. Jenkins read the second paper of the series, on "Commercially Useful Fluorescent Substances", which he dealt with under the headings : inorganic phosphors, fluorescent glass and fluorescent organic substances. A number of demonstrations were given to illustrate the luminescent characteristics of the various types of substances discussed.

The relation between the luminescent characteristics of the sulphide phosphors and the composition of the matrix, the activating metal and the furnacing treatment were briefly discussed. The most interesting property of the sulphides from the present point of view is their ability to fluoresce strongly when they are excited by near ultra-violet or even visible radiation.

Referring to the very beautiful colour effects obtained with these materials under filtered longwave ultra-violet radiation, Mr. Jenkins mentioned that luminescent sulphide paints were being used very effectively before the War in conjunction with black-glass lamps for fluorescent posters and displays of great beauty and artistic merit. The black-glass lamp comprises a quartz high-pressure mercury vapour discharge tube rated at 80 or $125 \mathrm{w}$. in an outer bulb of nickel glass. The transmission of the nickel glass at $3650 \mathrm{~A}$. is about 70 per cent and only a negligible amount of visible radiation is emitted by the lamp, which is one of the most convenient and efficient sources of near ultra-violet radiation available.

With normal paints and pigments, additive mixtures give subtractive mixtures of light. Thus a yellow pigment is yellow because it absorbs blue, and, as everyone knows, a mixture of blue and yellow paints gives green. In mixing fluorescent pigments, however, the artist has to deal with an additive mixture of lights, and he finds that blue and yellow no longer give green, but white. It is, of course, necessary for the artist to mix his luminescent pigments and to use them under ultra-violet radiation, with visible light excluded so far as possible. With comparatively little practice the new technique is easily acquired.

Some curious effects are observed with luminescent colours under conditions of scotopic vision; thus yellow luminescent inscriptions are more easily read than are blue, even when the brightness of the yellow is somewhat lower. This is probably due to the use of parafoveal vision for the shorter wave-lengths and consequent inability to focus. The high sensitivity of the periphery of the retina to blue and bluish-green colours makes it possible to see, although not to focus, these luminescent colours at brightness levels too low for normal vision to function. It can in certain circumstances produce a feeling of discomfort in the dark-adapted eye analogous to glare at normal illuminations.

Mr. Jenkins next described phosphors excited by ultra-violet radiation of wave-lengths shorter than $3000 \mathrm{~A}$., of which zine silicate, zine beryllium silicate and magnesium tungstate are perhaps the best known, 
and pointed out that these are commercially much more important than the sulphide types because of the efficiency with which they are excited by the low-pressure mercury vapour discharge. More than 50 per cent of the total input energy in the low. pressure mercury discharge is emitted as resonance radiation of wave-length $2537 \mathrm{~A}$. and below. It is possible to convert a considerable fraction of this energy into visible light by means of fluorescence, resulting not only in sources of high luminous efficiency, but also, what is scarcely less important, in sources in which the spectral luminosity can be controlled.

As might be expected, luminescence in glass is even more complex and difficult to understand than that in crystalline materials. The luminescence centre may be an ionized molecule such as $\mathrm{UO}_{2}++$, or a covalent molecule such as cadmium sulphide, or neutral atoms such as silver, lead and tin; or the luminescence may be due to a crystalline material such as zinc silicate precipitated in the glassy surround. In the latter case the glass is opalescent due to the presence of undissolved crystalline material. Mr. Jenkins referred to the various types and showed a number of examples. Apart from its use for discharge tubes, the fluorescence of glass activated by rare earth atoms has been used to study the structure of glass and to supplement the information given by $\mathrm{X}$-ray analysis.

Fluorescence has been used by glass manufacturers as a convenient method of identifying their product. Small amounts of cerium give a blue fluorescence and do not affect the colour or physical properties of the glass. Cerium has also been used as an indicator to study the movements of molten glass during manufacture.

Luminescent enamelled surfaces are usually prepared by binding certain sulphide phosphors in a boro-silicate type of enamel frit. By mixing suitable phosphors with certain synthetic moulding resins, luminescent plastics for use as switch covers, nameplates, etc., are prepared.

Referring to organic fluorescent materials, $\mathrm{Mr}$. Jenkins mentioned that commercial anthracene is a characteristic example. This substance fluoresces green in the solid state and blue in solution in benzene, and the green bands reappear if the benzene solution is frozen in liquid air. Very pure anthracene fluoresces blue in both the solid and solution states. It thus appears probable that the green fluorescence is caused by an impurity, stated to be naphthacene, in the crystalline anthracene matrix, and that the blue fluorescence is a property of the anthracene molecule itself.

Many organic compounds are fluorescent only in liquid or solid solution states, or when adsorbed at high dispersion on fibres or on inorganic adsorbents. The fluorescent dyestuffs such as fluorescein, eosin, rhodamine and auramine are well-known examples. Unlike the fluorescent dyestuffs, organic materials such as anthracene, quinine, etc., which fluoresce in the solid state, possess little or no body colour. Some of these colourless substances have all the characteristics of fluorescent dyes and can be incorporated in fabrics like any ordinary dye to give a colourless dyeing which is highly fluorescent. Dyes of this type have found an interesting application for marking linen in laundries, the sorting being done under black-glass lamps.

For spectacular lighting and display effects, fluorescent dyes are often used in conjunction with resins as lacquers and applied by brushing or spraying. When the lacquer is dry, the dye molecules are dis. persed in a thin film of resin which serves to isolate them energetically.

Two interesting engineering applications of organic fluorescent substances were mentioned. The first is in connexion with the surfacing of tools, gauges, etc., where the prussian blue used in the normal method is replaced by anthracene. The high spots appear as brightly fluorescing regions. The second application is to the detection of fine cracks in metals-particularly non-ferrous metals where the colloidal iron and powerful magnetic field method cannot be used. The metal part is treated with a strongly fluorescent substance, and after removal of this from the surface the cracks show up as fine bright lines under the black-glass lamp.

A paper on "Fluorescent Lamps: Their Photometric and Colour Rendering Properties and Measurement" was read by Mr. G. T. Winch. High-voltage fluorescent tubes, high-pressure mercury vapour fluorescent lamps and the newest types, the mainsvoltage fluorescent tubular lamps, were briefly reviewed. Mr. Winch described the accepted method of visual photometry when colour differences are involved : the field of view is kept down to about $2^{\circ}$ usually with a suitable filter in front of the sub standard tungsten lamp to minimize the colour difference. The transmission factor for the filter is determined from its spectral transmission and the luminosity distribution of the sub-standard lamp. Even when a colour match is achieved, it may be necessary to use a group of observers, owing to the large differences in luminosity distribution which generally exist.

Discussing physical photometric methods, Mr. Winch described his ingenious 'physical eye' photometer, where the light from the source after dispersal in a spectroscope passes through an aperture of special contour before falling on the photo-electric cell. By adjusting the contour of the mask, the apparatus is made to have 'average eye' spectral response without any knowledge of the spectral sensitivity of the photocell.

The difference between the colour of a source and its colour rendering was strikingly demonstrated by showing the wide difference in colour rendering of fabrics which could result when placed under lights of the same colour but of different spectral luminosity distribution. To assess the colour rendering properties of a source, it is necessary to know something about its luminosity distribution. It has been shown that for most purposes it is sufficient to measure the relative luminosity in about eight suitably chosen adjacent spectral bands. Much had already been accomplished in this connexion, but although the method showed considerable promise, the War has interrupted development work, and a good deal remains to be done before it can be applied to commercial fluorescent lamps.

Referring to the variability of natural daylight, $\mathrm{Mr}$. Winch pointed out that the colour temperature of the light from the sun varies over the enormous range of $2,000^{\circ}-25,000^{\circ} \mathrm{K}$. according to conditions. It is for this reason that industrial establishments concerned with colour rendering prefer, for their colour matching work, to use an artificial daylight source corresponding to one of the phases of daylight, usually that of a black-body radiator at a temperature of about $7,000^{\circ} \mathrm{K}$. The luminous efficiency of these artificial daylight sources is too low to make more 
than localized lighting with them possible, and it was not until the introduction of high-voltage fluorescent tubes that general lighting at high illuminations with a light of daylight quality became an economic possibility. The more convenient mains voltage fluorescent tube which was introduced later has made this form of lighting much more generally available.

Mr. Winch described how, by a process of repeated interchanges of lamps and measurements between the leading lamp manufacturers, and elaborate colour-rendering tests with many observers, the luminosity distribution of the present $5 \mathrm{ft}$., $80 \mathrm{w}$. fluorescent lamp was evolved. The colour of the tube is fairly close to that of illuminant $C$ specified by the C.I.E. $\left(6,500^{\circ} \mathrm{K}\right.$.), and the colour rendering is adequate for nearly all purposes except those concerned with the most critical discrimination of colour. The daylight colour of these lamps has proved of considerable psychological importance in war-time factories and other situations where natural daylight is largely or completely excluded and where work is carried on for twenty-four hours of the day.

Mr. F. W. Coppin read an interesting paper on "The Use of Fluorescent Pigments in Colour Printing". Owing to the impossibility of ensuring that the printing inks used in photomechanical colour reproduction processes have the theoretically correct hue, compensating corrections have to be made at some stage in the process. For relief printing these correctives take the form of local adjustments to the dot size in the plates, or 'fine etching' as it is called, and for photolithography, hand re-touching of the screen. These operations are highly. skilled and somewhat uncertain, and many attempts have been made to introduce the corrections by mechanical methods.

In the fluorescence process blue and green fluorescing materials are included in certain of the paints, and the colour separation negatives are made by mixing with the normal copying light some additional near ultra-violet radiation. The special paints comprise a set of eighteen water colours which are used by the artist under normal lighting conditions. Slight modifications in the normal technique are necessary to produce different hues by mixing colours, in order to avoid upsetting the fluorescence balance.

Arc illumination is used for making the colour separation negatives. For the yellow and magenta printing negatives, the arcs are shielded by special hoods fitted with filters which allow only ultra-violet, blue violet and green light to reach the copy. The correct ratio of ultra-violet to visible light is obtained by adjustable slits and compensating filters, using control patches on the copy-board. The blue-green printing negative is made using a panchromatic plate with the direct light of the arc on the copy and a Wratten No. 29 filter over the lens. For the black printing negative an infra-red sensitive plate is used and an infra-red filter over the lens.

Mr. Copping showed examples of colour charts prepared with the special paints and reproduced by the method described. For completely faithful reproduction, the hues of the four colour-printing inks should correspond to those for which the fluorescent paints have been balanced, but almost any good set of four colour inks will yield results which are sufficiently accurate for commercial purposes.

\section{OBITUARIES}

\section{Prof. Franz Boas}

Prof. Franz Boas, who died on December 21 last, was born at Minden, Westphalia, in 1858, and did most of his scientific work in North America, becoming instructor in anthropology at Clark University in 1882 after university studies at Heidelberg, Bonn and Kiel. He worked in physical anthropology, linguistics, evolution of material cultures and social characteristics and ceremonial, besides writing suggestive essays on questions of wide interest and making a notable attack upon the racist theories of Nazidom. Though he thus clearly belonged to what in Britain is sometimes called the HuxleyHaddon tradition of general practice in anthropology, he yet showed, as did Huxley and Haddon, a capacity for detailed observation as well as wide generalization. Among the outstanding features of his scientific work were his studies of the Eskimo in Baffin Land (1883-4) and his plan for and conduct of the Jessup North Pacific Expedition (1897 onwards) to study the relationships of peoples of North America and Northern Asia. As professor of anthropology in Columbia University (1899 onwards), as well as for a time eurator of ethnology at the American Museum of Natural History (190I-5), Boas found opportunities to co-ordinate the researches of different workers, and he became a central figure in the field of North American, and especially Eskimo, ethnology.

Boas's observations were much valued even if at times his theories were set aside; in such a field, theory is inevitably in a high degree provisional. $\mathrm{He}$ emphasized cultural similarities-the kayak, umiak, harpoon, household utensils, rituals and hero-tales, pictographic art and realistic carvings-between the Eskimo on one hand and the Chukchee and Koryak of north-east Asia on the other. But he rejected the view that the Eskimo peoples and their cultures had originated in Asia and spread to America. The findings of his Jessup expedition led him to think of the Eskimo of Alaska as comparatively new arrivals there from farther east in America. Indeed, he thought that several Palæ-Asiatic peoples of Siberia should be considered to have been immigrants from America. Needless to say, he shared the general view that the original peopling of America was due to movements from Asia; all he added to this was the idea of a backwash. Subsequent work has revealed evidence of older cultures in Alaska; and it is increasingly realized that diffusions of culture and movements of peoples in aretic America and the region around the Bering Sea have been complex, and probably by no means all in one direction; but ancient drifts from Asia to America are universally thought to have been fundamentally important. An Asiatic cradle for Eskimo culture is nevertheless not generally accepted, and Stefansson, for example, has the view that from the forests north of Lake Superior the ancestors of the Eskimo drifted northwards to the Barren lands and thence went on both eastwards to Greenland and westwards to Alaska. It seems established that the arctic Eskimo culture, which has an obvious unity throughout, came into the Arctic two thousand or more years ago, and was once more prosperous than it has been in recent centuries.

Boas believed that the peoples of the extreme south and south-east of South America and those of the north-west of North America must be considered fringing groups imperfectly imbued with the culture 